\title{
Eleonora de Fonseca Pimentel, le monitore napoletano et le problème de la participation politique
}

Anna-Maria Rao

\section{(2) OpenEdition \\ 10 Journals}

\section{Édition électronique}

URL : https://journals.openedition.org/ahrf/6363

DOI : 10.4000/ahrf.6363

ISSN : 1952-403X

Éditeur :

Armand Colin, Société des études robespierristes

\section{Édition imprimée}

Date de publication : 1 juin 2006

Pagination : 179-191

ISSN : 0003-4436

\section{Référence électronique}

Anna-Maria Rao, «Eleonora de Fonseca Pimentel, le monitore napoletano et le problème de la participation politique », Annales historiques de la Révolution française [En ligne], 344 | avril-juin 2006, mis en ligne le 01 juin 2009, consulté le 24 avril 2022. URL : http://journals.openedition.org/ahrf/6363 ; DOI : https://doi.org/10.4000/ahrf.6363

Ce document a été généré automatiquement le 24 avril 2022.

Tous droits réservés 


\title{
Eleonora de Fonseca Pimentel, le monitore napoletano et le problème de la participation politique
}

\author{
Anna-Maria Rao
}

1 En évoquant les insurrections anti-républicaines dans les provinces du ci-devant Royaume de Naples, Vincenzo Cuoco, dans son Histoire de la révolution de Naples, rappelait un des célèbres articles parus dans le Monitore napoletano où Eleonora de Fonseca Pimentel avait abordé la question : «Il terrorismo cogl'insorgenti si provò sempre inutile. "E che?" scrivea la saggia e sventurata Pimentel " quando un metodo di cura non riesce, non se ne saprà tentare un altro? $»^{1}$.

Dans sa traduction du livre de Cuoco publiée en 1807, Bertrand Barère traduisait ainsi ce passage : «Le terrorisme fut toujours sans effet sur les insurgés. Eh quoi, écrivait le sage et infortuné Pimentel, quand un remède ne réussit pas, n'en saurait-on essayer un autre ? $»^{2}$. Faute d'écriture, malentendu ? Sous la plume de Barère, Eleonora devient «le sage et infortuné Pimentel ». Mais lorsque Cuoco rappelle de nouveau la rédactrice du Monitore, dans le chapitre dédié à l'évocation émue et touchante de "Quelques patriotes " péris sur l'échafaud, ici Barère ne se trompe plus, et comme pour compenser le lapsus précédent, dans une traduction qui est pour le reste assez fidèle, il ajoute " célèbre » là où Cuoco n'avait écrit que « cette femme »:

«ELEONORE FonSECA PIMENTEL. Audet viris concurrere virgo. Mais elle fut poussée vers la Révolution, comme Camille vers la guerre, par le seul amour de la patrie. Jeune encore, les vers de cette femme célèbre avaient mérité l'approbation de Métastase. Mais la poésie ne faisait que la moindre partie des connaissances dont son esprit était orné. Pendant la durée de la république, elle rédigea le Moniteur républicain, qui respirait le plus pur et le plus ardent amour de la patrie. Cette feuille lui coûta la vie, et elle affronta la mort avec une indifférence égale à son courage. Avant de marcher vers l'échafaud, elle demanda du café : forsan haec olim meminisse juvabit, furent ses dernières paroles. $»^{3}$

L'image d'Eleonora de Fonseca Pimentel en héroïne classique - peut-être encore inconnue en France, malgré l'attention prêtée par la presse française aux événements 
de Naples ${ }^{4}$ - commençait ainsi à faire le tour de l'Europe grâce aux écrits des patriotes napolitains bannis de l'Italie depuis 1799, en exil en France d'abord et, après Marengo, dans la République cisalpine.

Avant la parution de la traduction française de l'ouvrage de Cuoco, Helen Maria Williams avait tracé la première histoire anglaise de la République napolitaine, dans le cadre de son Aperçu général de l'état de la France et des républiques sœurs en 1799, publié en anglais en 1801 et tout de suite traduit en français ${ }^{5}$. Pour sa reconstruction des événements de Naples elle se servait d'un mémoire qui lui avait été fourni par le patriote Amedeo Ricciardi, exilé à Paris ${ }^{6}$. Là aussi se trouvait le martyrologe des patriotes conduits à la mort, parmi lesquels Eleonora Pimentel, dont elle écrivait: "Eléonora Fonseca, qui unissait les grâces de son sexe à un génie supérieur, eut la gloire de partager leur sort; ses connaissances littéraires lui avoient mérité une haute considération : elle fixa dans sa plus tendre jeunesse les regards de $\mathrm{M}$. de Voltaire, qui en parle avec éloge $~$. Elle omettait la référence à la rédaction du Monitore qui, dans le manuscrit de Ricciardi, ne manquait pourtant pas ${ }^{8}$. Et elle concluait le chapitre de son Aperçu dédié à «l'état des femmes dans la Révolution française» en rappelant l'exemple des femmes de Naples, considérées en modèle de générosité et de courage, d'héroïsme et de dévotion à la République. Elle en profitait pour se demander, non pas si les femmes avaient obtenu quelque chose de la Révolution, mais si elles avaient obtenu autant qu'elles auraient dû̀ .

Grâce et génie philosophique, relations littéraires et mort héroïque: ces traits se figèrent dans une image durable, reçue et transmise par la littérature jusqu'à nos jours. Même dans l'historiographie, cette image a souvent gardé sa force d'attraction.

On doit à Benedetto Croce, dès 1887, la première biographie de la rédactrice du Monitore, fondée sur une documentation qui n'a guère été enrichie par la suite ${ }^{10}$. Il soulignait, lui aussi, le " noble caractère d'une femme toujours en première ligne dans les batailles intellectuelles et politiques de son âge», l'énergie et la "chaleur de sentiment " d'une femme qui, toutefois, ne pouvait pas être considérée parmi «les penseurs et les chercheurs originaux qui occupent une place dans l'histoire d'une science ou d'une discipline spécifique $»^{11}$.

D'autres sont revenus par la suite sur sa vie privée et sur ses ouvrages littéraires, pour essayer de la soustraire au sort historiographique de la galerie des " femmes célèbres ", ou bien pour retrouver dans ses écrits les traces d'un engagement féministe ${ }^{12}$. Franco Venturi a tracé un portrait, bref mais très dense, de l'« estrangeirada » portugaise dans le milieu intellectuel et politique des réformes de Pombal ${ }^{13}$. Les romans d'Enzo Striano et de Maria Antonietta Macciocchi, traduits aussi en français, ont contribué à la divulgation de sa biographie auprès d'un public plus large ${ }^{14}$.

Mais il convient de rappeler encore une fois quelques données de sa biographie intellectuelle ${ }^{15}$, avant d'aborder le rôle qu'elle exerça en 1799 dans le journalisme et dans la vie politique de la République napolitaine.

9 Sa famille vivait à Rome, où elle naquit en 1752, quand elle fut obligée en 1760 de quitter la ville, à la suite des tensions entre le Portugal et la papauté provoquées par l'expulsion des Jésuites de la part de Pombal. Elle se transféra à Naples où, dès la fin des années 1760, Eleonora se faisait remarquer par sa production poétique dans les milieux académiques et littéraires. En 1768 elle faisait déjà partie de l'Académie des Philalètes et de l'Arcadie et participait aux réunions du salon littéraire des frères De Gennaro. En 
1770 elle entrait en correspondance avec le grand poète Pietro Metastasio, protagoniste reconnu de la République des lettres ${ }^{16}$. En 1776 elle échangeait des vers avec Voltaire dans la revue Giornale letterario di Siena ${ }^{17}$.

10 Croce ne vit rien d'autre dans sa production poétique - des sonnets pour le mariage de Ferdinand IV et Marie Caroline et pour la naissance de leurs enfants, pour d'autres mariages encore et des décès, des cantates, des oratorios - qu'une production courtisane et d'occasion ${ }^{18}$. Mais il n'y avait pas que cela. Sa cantate Le Triomphe de la vertu, publiée en 1777 à la suite d'un attentat à la vie du ministre Pombal, était précédée par une lettre-dédicace au ministre portugais qui était une sorte de manifeste du modèle du prince éclairé, engagé dans les réformes nécessaires au bonheur public, et qui célébrait son action pour la justice et contre l'esclavage, pour la promotion des sciences, de la culture, de l'éducation ${ }^{19}$. Un programme que partageaient les milieux réformateurs de Naples dans la période 1770-1780, et que les frères Grimaldi, Giuseppe Maria Galanti, Mario Pagano, Gaetano Filangieri, énonçaient dans des ouvrages d'histoire, d'économie, de législation. Eleonora de Fonseca, comme Ignazio Ciaia, Antonio Jerocades, Antonio Di Gennaro duca di Belforte et d'autres, poursuivaient le même but par le langage poétique.

11 Le langage est en effet une des clefs les plus importantes pour la compréhension de la place occupée par de Fonseca dans la vie intellectuelle et politique napolitaine des réformes à la Révolution. La poésie n'était pas du tout une production purement académique mais, comme le théâtre, elle était une forme de communication, qui aspirait aussi à un statut disciplinaire. Pendant les années 1780, au moment même où le mouvement réformateur à Naples assume un rôle fondamental dans la vie politique de la monarchie, on assiste simultanément à une pression accrue des intellectuels pour faire reconnaître leur rôle professionnel et revendiquer la possibilité de vivre de leur plume $^{20}$. Et d'autre part, si la fondation de l'Académie des Sciences en 1778 avait ouvert quelques débouchés aux hommes de la République des lettres ${ }^{21}$, l'Arcadie restait traditionnellement l'académie la plus ouverte aux femmes pour qui, tout au long du XVIII siècle, la poésie resta un des moyens principaux de "prise de parole publique ». En percevant avec clarté les changements en cours dans le goût du public des lecteurs, et des lectrices ${ }^{22}$, Eleonora de Fonseca assignait à la littérature une fonction éminemment civile et politique. Elle écrivit en 1799, dans son Monitore napoletano, à propos de l'édition napolitaine du Candide: «Voltaire avec des petits romans répandait toujours des germes de philosophie, de liberté, d'égalité $»^{23}$.

"Femme de lettres », donc, mais aussi de science, comme le montrent non seulement l'éloge des mathématiques dans son Triomphe de la vertu, mais aussi ses correspondances, en particulier les lettres échangées avec Alberto Fortis, homme de science de Vérone, lié aux milieux intellectuels du Royaume de Naples qu'il visita dans les années 1780 .

Ce sont justement ses relations intellectuelles que lui reprocha son mari Pasquale Tria, un militaire brutal qu'elle avait épousé en 1777, qui la contraignit à s'occuper de ses sœurs, qui osa amener chez eux sa maîtresse et qui, après qu'elle eût déjà perdu en 1779 son premier enfant, la battit jusqu'à la faire avorter. Pendant le procès de séparation, son mari lui reprocha précisément d'avoir négligé ses devoirs de femme pour ses études. Privée d'argent pour s'acheter des livres, elle avait même joué au loto pour s'en procurer : et grâce à un numéro gagnant elle avait pu s'abonner à l'Encyclopédie ${ }^{24}$. 
14 Elle a vécu donc jusqu'au drame le conflit entre famille et études. Grâce au soutien de son père elle réussit en 1785 à se séparer de son mari, et s'adonna alors entièrement aux livres et à l'écriture de textes poétiques ou en prose, où se retrouvent quelques idées force constantes : la célébration de la liberté, l'exaltation d'un modèle de royauté éclairée, la défense de la juridiction de l'État contre celle de l'Église, la défense de l'indépendance de la «nation napolitaine ». En 1789, avec Antonio Jerocades, Francesco Salfi, et plusieurs autres, elle contribue à un recueil de compositions poétiques qui célèbrent l'inauguration à San Leucio, près de Caserte, d'une manufacture royale de soieries accompagnée d'une législation sociale et communautaire pour les ouvriers ${ }^{25}$. L'année suivante elle traduit en italien le livre de Niccolò Caravita, Nullum ius pontificis maximi in regno neapolitano, publié en latin en 1707, dédiant sa traduction au roi de Naples. Encore une fois, il s'agit pour elle de proposer au souverain une pratique de gouvernement orientée vers le bonheur public. Elle rappellait le long cheminement parcouru pour arriver finalement à supprimer, en 1788, l'hommage de l'haquenée, symbole de la dépendance féodale du Royaume de Naples envers la papauté qui, en vain, avait été niée dès le début du siècle par Caravita et par Pietro Giannone. Celui-ci, écrivait-elle, «illustre champion et martyr de la cause nationale », par ses écrits avait " presque fait de nous une nation nouvelle». Surtout, elle s'interrogeait sur la nature du droit public et des fondements de l'État, ce qui l'amenait à suggérer une théorie de l'impôt fondée sur la souveraineté populaire. Les peuples ne pouvaient pas être traités comme des fiefs, transmis d'un souverain à l'autre comme s'ils relevaient du droit privé. Le droit public ne pouvait se baser que «sur la nature et sur les droits de l'homme »:

$$
\begin{aligned}
& \text { «Le royaume n'est pas un patronage, n'est pas un héritage de primogéniture, n'est } \\
& \text { pas un fidéicommis : le royaume est administration et défense des droits publics de } \\
& \text { la nation, conservation et défense des droits privés de chaque citoyen. Pour cette } \\
& \text { administration et pour cette conservation il faut des lois, donc la faculté législative } \\
& \text { dans le prince ; pour ces forces il faut des revenus, donc les tributs au prince; et les } \\
& \text { tributs ont donc une mesure relative et proportionnelle aux besoins de la Nation, } \\
& \text { qui ne sont pas toujours les mêmes. »"6 }
\end{aligned}
$$

Mais le modèle du prince éclairé révélait désormais ses ambiguïtés et son caractère illusoire face aux exigences constitutionnelles des réformateurs. La Révolution française venait de montrer un autre modèle politique, une autre possibilité d'action. Et ce fut, à Naples, le temps des conspirations et de la répression. Arrêtée en octobre 1798, Pimentel fut libérée en janvier 1799, à l'approche de l'armée du général Championnet. Elle participa ainsi à la prise du château de Saint Elme, où la République napolitaine fut proclamée le 21 janvier. Dès le 2 février, le premier numéro du Monitore napoletano sortait. C'était la naissance du journalisme politique à Naples et ce fut Eleonora qui rédigea le journal le plus important de la République napolitaine, un des principaux parmi les nombreux titres qui parurent dans l'Italie en révolution: deux fois par semaine, le mardi et le samedi, pour un total de 35 numéros de quatre pages chacun, infolio, jusqu'au dernier numéro, paru le 8 juin. Le 13 juin, l'armée "sanfédiste » du cardinal Ruffo était à Naples. Condamnée à mort le 17 août, Eleonora de Fonseca Pimentel fut conduite à l'échafaud le 20 août 1799 avec d'autres patriotes, les ci-devant nobles Giuliano Colonna et Gennaro Serra, l'évêque Michele Natale, l'avocat Vincenzo Lupo, le prêtre et professeur de botanique Nicola Pacifico, les banquiers Domenico et Antonio Piatti. Elle et Luisa Sanfelice ${ }^{27}$, exécutée le 11 septembre 1800, furent les seules femmes condamnées à mort. 

$\mathrm{du}$ Monitore napoletano ${ }^{28}$. Faute de documents, une longue tradition historiographique, de Benedetto Croce à Mario Battaglini, lui a attribué la plupart, sinon la totalité, des articles publiés dans ce journal.

on retrouve dans le Monitore les mêmes soucis et les mêmes idées-force qu'elle avait déjà exprimés plusieurs fois. L'effort de communication, avant tout, la simplicité et la force du style ${ }^{29}$. Mais aussi et surtout, la défense de la liberté et de l'indépendance du nouvel État républicain dans ses rapports avec la France, d'où la nécessité, plusieurs fois affirmée, d'une structure militaire autonome ; l'égalité des citoyens de la nouvelle nation, d'où ses polémiques contre le projet de réintroduire dans l'armée un corps de chevalerie réservé à la noblesse; enfin, la nécessité de poser le peuple et la souveraineté populaire comme fondement du nouvel État.

C'est surtout ce dernier aspect qui constitue le véritable fil conducteur de ses articles, le thème central autour duquel tous les autres s'organisent et se structurent. Dès le 9 février, dans un article célèbre, elle pose le problème de «qu'est-ce qu'un peuple », et en particulier le peuple de Naples :

«Cette partie du peuple qu'il faudra, jusqu'à ce qu'une meilleure instruction ne l'élève à la vraie dignité de Peuple, continuer à appeler plèbe, comprend non seulement la nombreuse population de la ville, mais aussi la population plus respectable des campagnes; et si dans les monarchies la force de l'état repose sur cette partie du peuple, dans la démocratie s'y appuie non seulement la force mais aussi la dignité de l'État. Une grande ligne de séparation, peut-être majeure chez nous que partout ailleurs, divise parmi nous cette partie du reste du peuple, justement parce que nous n'avons pas avec elle un langage commun. Si l'on remonte à l'origine de nos malheurs on verra qu'ils sont provoqués particulièrement par cette séparation [...] Tant que l'éducation nationale n'aura pas transformé la plèbe en un Peuple, il faut que le Peuple s'adapte à parler comme la plèbe $[. .$.$] à se mélanger avec elle. »^{30}$

Patriotes, peuple et plèbe : elle posait d'emblée la question cruciale du passage des Lumières à la Révolution dont elle avait eu déjà une conscience aiguë dans les années précédentes, s'apercevant que le mouvement des Lumières ne faisait qu'accroître la distance entre le public cultivé et ceux qui ne savaient ni lire ni écrire, les «deux peuples" divisés "par deux siècles et par deux degrés de température " écrira Vincenzo $\mathrm{Cuoco}^{31}$.

D'où l'importance de l'« éducation nationale » comme moyen d'intégration nationale et républicaine ${ }^{32}$. Mais plusieurs aspects distinguent nettement la position d'Eleonora Fonseca Pimentel des autres patriotes qui insistaient sur ce même thème. En premier lieu, elle posait le problème de l'éducation nationale non seulement sur un plan théorique mais sur un plan éminemment pratique. «Je rappelle - écrivait-elle le 5 mars - à nos dignes Représentants qu'il n'est pas seulement utile mais qu'il est un devoir dans une démocratie que le peuple soit à connaissance des faits et qu'il puisse en juger : si non, comment pourra-t-il y prendre intérêt? L'instruction théorique peut faire quelques philosophes, la seule instruction pratique fait une nation. $»^{33}$

Il en découlait, encore une fois, la question du langage et des moyens de communication à adopter pour répandre les principes républicains. Elle propose donc la rédaction de journaux en dialecte, non pas, évidemment, pour que les analphabètes pussent les lire, mais pour en effectuer la lecture à haute voix par des intermédiaires culturels $^{34}$. Cette mesure a été jugée ingénue et inutile car la composition patriotique en 
dialecte gardait un style rhétorique cultivé bien éloigné de l'outillage culturel populaire ${ }^{35}$. Le débat sur cette question ne peut que rester largement ouvert; mais l'on pourrait rappeler que, grâce non seulement au théâtre ${ }^{36}$, mais aussi au barreau, qui occupait une place centrale dans la vie sociale et culturelle de la ville de Naples, la rhétorique classique était peut-être beaucoup plus familière que l'on ne le pense d'habitude.

Le théâtre était donc l'un des instruments envisagés par le Monitore comme moyen de communication et d'éducation, en particulier le théâtre des marionnettes. Et, encore, les catéchismes républicains, les romans, les affiches dans les quartiers les plus populaires de la ville, pour donner de la publicité aux actes du gouvernement.

Mais Eleonora de Fonseca Pimentel identifiait les moyens principaux de l'éducation républicaine dans la législation et dans la participation politique. Pour cette raison elle s'opposa toujours aux punitions sommaires des insurgés antirépublicains dans les provinces : il fallait punir les individus non pas les communautés ${ }^{37}$; et surtout fallait-il émaner des lois, comme l'abolition de la féodalité, qui montraient que le gouvernement républicain était " plus utile aux Peuples $»^{38}$.

Quant à la participation politique, elle en considérait les différentes voies. Les fêtes républicaines et les salles d'instruction publique, bien sûr, pour lesquelles elle montre une sensibilité particulière au contraire de Cuoco, par exemple, qui manifeste du mépris à l'égard des salles d'instruction, considérées comme des lieux de fainéantise. Eleonora de Fonseca Pimentel en suit constamment les activités, elle en publie les discours, elle consigne la participation du peuple du marché et des femmes ; elle-même y participe, en récitant un Hymne à la liberté3 ${ }^{39}$.

Elle soutient l'importance du droit de pétition, «commun à chaque individu dans la démocratie ", et elle le pratique à propos du choix de l'emblème de la République napolitaine. L'emblème choisi par le gouvernement provisoire était semblable à celui de la France : une femme à demi nue avec le faisceau consulaire et la pique surmontée par le bonnet rouge. Elle propose de le remplacer par une enseigne capable de rappeler les maximes fondamentales de la République, la justice, les vertus civiques : et donc, en renversant l'imaginaire et la symbolique du genre, son emblème représente un " homme robuste debout ", d'un âge compris entre la " jeunesse et la virilité », habillé à la romaine d'une toge bleue, avec l'épée d'un côté, la bêche de l'autre, le pied droit repoussant les emblèmes de la richesse, en haut un génie régissant la balance de la justice, dans la campagne sur le fond des instruments d'agriculture et de guerre ${ }^{40}$.

Mais elle n'envisage pas seulement les nouveautés républicaines comme moyens d'éducation et de participation politiques. Les traditions religieuses et les peurs populaires peuvent servir aussi en l'occurrence. Ainsi le miracle de Saint Janvier qui avait suivi l'arrivée de Championnet à Naples: elle reproche aux patriotes de ne pas avoir tiré tous les avantages qu'il fallait de cette occasion pour réaliser la "réconciliation du menu peuple avec le nouveau système». On aurait dû mieux souligner l'«évidente décision du Ciel en faveur de la République». On aurait dû souligner qu'il avait plu pendant tout le temps de l'expédition de Ferdinand IV contre la République romaine, tandis qu'il avait fait beau durant la marche des Français vers Naples. De même, aurait-on dû remarquer la petite éruption du Vésuve comme signe de joie et d'illumination après la proclamation de la République ${ }^{41}$.

Elle donnait ainsi une leçon de pragmatisme et de réalisme en face d'un laïcisme qui se battait de façon abstraitement rigoureuse contre ce qu'il condamnait comme 
superstition. Enfin, elle abordait aussi le droit de vote, un des problèmes majeurs du «triennio " républicain italien puisque la sujétion à la tutelle française ne permettait pas des libres élections. Elle suggérait donc au gouvernement provisoire de participer à la procession du Corpus Domini, avec les représentants des municipalités républicaines des cantons et avec des députations des pères de famille, choisis dans les arts des différents districts ; les femmes du menu peuple devraient être accueillies sur la scène : "ainsi le Peuple sentira dans les faits d'être plus considéré qu'auparavant; il commencera à goûter le plaisir d'élire, l'honneur d'être élu [...] il s'habituera petit à petit aux assemblées primaires, c'est-à-dire aux augustes fonctions de citoyen. $»^{42}$

Le débat historiographique récent a parfois eu tendance à séparer, parfois même à opposer l'exercice du droit de vote d'un côté, et la sociabilité politique de l'autre, comme voies d'apprentissage de la citoyenneté. Dans le cas d'Eleonora de Fonseca Pimentel il est évident qu'il n'y avait aucune opposition ni séparation mais, au contraire, un lien étroit entre droit d'association, salles d'instruction et élections. D'autres patriotes napolitains, comme Cuoco, Vincenzo Russo ou Mario Pagano, considéraient qu'il fallait attendre qu'un long processus d'éducation soit accompli avant que le peuple pût commencer à exercer ses droits politiques. Dans son projet de constitution, Mario Pagano posait la capacité de lire et écrire et la connaissance du catéchisme républicain parmi les conditions de la citoyenneté active ${ }^{43}$. Eleonora de Fonseca Pimentel, au contraire, se battait dans son Monitore pour la mise en œuvre immédiate de tous les moyens possibles d'apprentissage et de pratique de la démocratie.

On a remarqué dans son journal l'absence d'une prise de position en faveur de la participation politique des femmes ${ }^{44}$; absence d'autant plus significative au vu de l'importance du thème général de la participation politique dans ses articles, en ce qui concerne les rapports entre les patriotes locaux et la France comme en ce qui concerne les rapports entre les patriotes et les masses populaires. Oubli, refoulement, passage de la théorie à l'action? Ce silence partiel dépendait peut-être de ce même sens du concret et de l'immédiat qui orientait sa réflexion politique. Parmi les patriotes qui avaient participé à la prise de Saint Elme, plusieurs firent partie du gouvernement provisoire et des commissions qui furent par la suite nommées par le général Macdonald, tandis que Pimentel s'adonna au journalisme. Son activité de journaliste fut sûrement le fruit d'un choix cohérent avec son activité précédente; mais aussi le fruit d'une exclusion des charges publiques que pratiquement personne n'envisageait encore comme possible pour une femme. Elle préféra ne pas aborder explicitement le problème de cette exclusion en montrant par son activité l'inexistence de tout argument pour la justifier ; elle voua toutes ses forces à indiquer les voies et les mesures nécessaires aptes à intégrer le peuple tout entier - femmes comprises - dans la république et dans la citoyenneté. 


\section{NOTES}

1.Parmi les nombreuses éditions de l'ouvrage de Cuoco, récemment traduit en français (Vincenzo Cuoco, Essai historique sur la révolution de Naples, édition bilingue, texte établi par Antonino DE FRANCESCO, introduction, traduction et notes de Alain PONS, préface de Michel Vovelle, Paris, Les Belles Lettres, 2004), je cite de l'édition Vincenzo Cuoco, Saggio storico sulla rivoluzione di Napoli, introduzione di Pasquale Villani, Milano, BUR, 1999, p. 241-242.

2.Vincenzo Cuoco, Histoire de la Révolution de Naples, ristampa anastatica della traduzione di Bertrand Barère (1807), a cura di Anna Maria Rao e Maïté BouYsSY, Napoli, Vivarium, 2001, p. 307. Sur les fautes, les variations ou les malentendus de la traduction de Barère, je renvoie à Anna Maria Rao, «Bertrand Barère e Vincenzo Cuoco. Come uscire dalla rivoluzione », ibid., p. 9-57 et Maïté BouYssy, « Vincenzo Cuoco et Bertrand Barère. Regards croisés pour révolutions perdues ", ibid., p. 59-108.

3.Vincenzo Cuoco, Histoire de la Révolution de Naples, op. cit., p. 403 et cf. Anna Maria RAO, «Bertrand Barère e Vincenzo Cuoco », op. cit., p. 42. Ainsi le texte original : Pimentel Eleonora Fonseca. Audet viris concurrere virgo. Ma essa si spinse nella rivoluzione, come Camilla nella guerra, per solo amor della patria. Giovinetta ancora, questa donna avea meritata l'approvazione di Metastasio per i suoi versi. Ma la poesia formava una piccola parte delle tante cognizioni che l'adornavano. Nell'epoca della repubblica scrisse il Monitore napoletano, da cui spira il più puro ed il più ardente amor di patria. Questo foglio le costò la vita, ed essa affrontò la morte con un'indifferenza eguale al suo coraggio. Prima di avviarsi al patibolo, volle bevere il caffè, e le sue parole furono : « Forsan haec olim meminisse juvabit " (Vincenzo Cuoco, Saggio storico, op. cit., p. 307). 4.La Décade philosophique, par exemple, le 30 thermidor an VIII, avait déploré la mort " des personnes les plus connues, qui périssent sur l'échafaud » et condamné la répression voulue par la reine de Naples qui « ne pardonne ni au sexe, ni à l'âge, ni au talent ", mais sans évoquer le nom de Eleonora Pimentel. Cf. Anna Maria RAo, Esuli. L'emigrazione politica italiana in Francia (1792-1802), Napoli, Guida, 1992, p. 253. Sur la République napolitaine dans la presse française, cf. aussi Anna Maria RAO, La Repubblica napoletana del 1799 nella stampa periodica francese, Napoli, La Città del sole, 1999 ; Michèle BENAITEAU, «Tra informazione e ideologia : la rivoluzione napoletana del 1799 nella stampa periodica francese », dans Augusto Placanica, Maria Rosaria Pelizzari (dir.), Novantanove in idea. Linguaggi, miti, memorie, Napoli, Edizioni scientifiche italiane, 2002, p. 595-615.

5.Sketches of the State of Manners and Opinions in the French Republic, Towards the Close of the Eighteenth Century, in a Series of Letters, London, G.G. and J. RoBinson, 1801, 2 vol. ; Aperçu de l'état des mours et des opinions dans la République française, vers la fin du XVIII siècle; par Hélène-Maria WiLliams, traduit de l'anglais par Madame [Sophie] Grandchamp, Paris, chez les frères Levrault, quai Malaquais, Strasbourg, chez les mêmes, an IX (1801), 2 vol. La préface à l'édition française est datée de Paris, le 20 vendémiaire IX (12 octobre 1800). Cf. Anna Maria RAO, Tra civiltà e barbarie : storie inglesi della Repubblica napoletana del 1799, dans Renata de LORENzo (dir.), Risorgimento, democrazia, Mezzogiorno d'Italia, Studi in onore di Alfonso Scirocco, Milano, Franco Angeli, 2003, p. 708-739. 
6. Benedetto MARESCA, « Memoria sugli avvenimenti di Napoli nell'anno 1799 scritta da Amedeo Ricciardi napoletano », Archivio storico per le province napoletane, XIII, 1888, p. 36-94. Cf. à présent Amedeo RICCIARDI, Napoli 1799. Memoria sugli avvenimenti, a cura di Silvana Musella, Napoli, Franco Di Mauro, 1994.

7.Aperçu de l'état des mouurs, op. cit., I, p. 189.

8.Amedeo RICCIARDI, Napoli 1799, op. cit., p. 106 : « Eleonora Fonseca, donna che riuniva alle grazie di Saffo la filosofia di Platone. Avea ella meritati i segni della stima di Voltaire, e fu a tempo della Repubblica la redattrice del Monitore ».

9.Cf. Anna Maria RAO, Tra civiltà e barbarie, op. cit., p. 726.

10. Benedetto CROCE, La rivoluzione napoletana del 1799. Biografie - Racconti - Ricerche, Bari, Laterza, 1968 (1ère éd. 1887-1896), p. 3-83. On doit également à Croce la première édition partielle du Monitore napoletano, publiée en 1943.

11.Benedetto $\mathrm{C}_{\mathrm{ROCE}}$, La rivoluzione napoletana, op. cit., p. 3.

12.Franco SCHIATTARELlA, La marchesa giacobina. Eleonora Fonseca Pimentel, Napoli, Schettini, 1973 ; Annarita ButTAFuoco, « Eleonora Fonseca Pimentel : una donna nella rivoluzione ", Donna, Woman, Femme, 3, aprile-giugno 1977, p. 51-92 ; Marisa SARACINELLI, «Eleonora Fonseca Pimentel: tra rivoluzione e diritti delle donne », dans Ivana RICCI (dir.), Senza camelie ; percorsi femminili nella storia, Ravenna, Longo, 1992, p. 13-27 ; Laura Capobianco, Cesira D'agostino, Anna de Mascellis, Rosetta Gervasio, Adriana Pantani, « Una giacobina donna : Eleonora Pimentel Fonseca », dans Laura CAPOBIANCO (dir.), Donne tra memoria e storia, Napoli, Liguori, 1993, p. 133-162 ; Mario BATTAGLINI (dir.), Eleonora Fonseca Pimentel. Il fascino di una donna impegnata fra letteratura e rivoluzione, Napoli, Procaccini, 1998 ; Elena URGNANI, La vicenda letteraria e politica di Eleonora de Fonseca Pimentel, prefazione di Luisa MURARo, Napoli, La Città del Sole, 1998 ; Eleonora de Fonseca Pimentel, Sonetti in morte del figlio, Napoli, La Città del Sole, 1999 ; Teresa SANTos, Sara Marques Pereira (dir.), Leonor da Fonseca Pimentel. A portuguesa de Nápoles (1752-1799), Lisboa, Livros Horizonte, 2001.

13.Franco VENTURI, Settecento riformatore, vol. IV, La caduta dell'Antico Regime (1776-1789), t. I : I grandi stati dell'occidente, Torino, Einaudi, 1984, p. 227-230, 235, 238.

14.Enzo StRIANo, Il resto di niente, Napoli, Loffredo, 1986, Cava de' Tirreni, Avagliano, 1997, trad. fr. de Jérôme NicolAs, Rien de rien, Paris, Phébus, 2002 ; Maria Antonietta MAcCiocchI, Cara Eleonora. Passione e morte della Fonseca Pimentel nella rivoluzione napoletana, Milano, Rizzoli, 1993, trad. fr. de Monique BACCELLI, Eleonora : la vie passionnée d'Eleonora Fonseca Pimentel dans la Révolution napolitaine, Paris, éd. du Félin, 1995.

15.Appartenant, sauf indications contraires, aux ouvrages déjà cités de Croce et Venturi.

16.Cf. Mario VALENTE (dir.), Legge poesia e mito. Giannone Metastasio e Vico fra " tradizione » $e$ « trasgressione » nella Napoli degli anni Venti del Settecento, saggio introduttivo di Giuseppe Galasso, Roma, Aracne, 2001.

17.La revue publiait en juillet 1776 ces Versi del sig. Di Voltaire responsivi ad un sonetto della nobile ed egregia donzella Eleonora Fonseca di Pimentel abitante a Napoli : «Beau rossignol, de la belle Italie/votre sonnet cajole un vieux hibou/au mont Jura retiré dans un trou/sans voix, sans plumes et privé de génie./ Il veut quitter son païs morfondu:/ auprès de vous à Naples il va se rendre;/s'il peut vous voir et s'il peut vous entendre/ il reprendra tout ce qu'il a perdu ». Franco Venturi, op. cit., p. 228 n. Sur la revue, cf. Renato PASTA, « Il " Giornale letterario di Siena " (1776-1777) ed i suoi collaboratori », Rassegna storica toscana, XXIV, 1978, p. 93-135. 
18. Benedetto CROCE, op. cit., p. 10. Ces ouvrages sont à présent reproduits dans Elena URGNANI, La vicenda letteraria e politica, op. cit.

19.Benedetto CROCE, op. cit., p. 12-13 ; Franco VENTURI, op. cit., p. 228-230 ; Elsa Rita Dos SANTOS, «"O triunfo da virtude" (1777) », dans Teresa SANTOS, Sara MARQUES PEREIRA (dir.), Leonor da Fonseca Pimentel. A portuguesa de Nápoles, op. cit., p. 139-151. 20.Cf. Anna Maria RAO, «Intellettuali e professioni a Napoli nel Settecento ", dans Maria Luisa BETRI, Alessandro PASTORE (dir.), Avvocati, medici, ingegneri. Alle origini delle professioni moderne, Bologna, Clueb, 1997, p. 41-60 ; Id., " Illuminismo e massoneria: Antonio Jerocades nella cultura napoletana del Settecento ", dans Antonio Coco (dir.), Le passioni dello storico. Studi in onore di Giuseppe Giarrizzo, Roma, Edizioni del Prisma, 1999, p. 481-510.

21.Elvira CHIOSI, “ “Humanitates” e scienze. La Reale Accademia napoletana di Ferdinando IV : storia di un progetto », Studi storici, XXX, 1989, p. 435-456; Id., Lo spirito del secolo. Politica e religione a Napoli nell'età dell'illuminismo, Napoli, Giannini, 1992, p. 107-142 ; Id., « Lo Stato e le scienze. L'esperienza napoletana nella seconda metà del Settecento ", dans Giulio BARSANTI, Vieri BECAGLI, Renato PASTA (dir.), La politica della scienza. Toscana e stati italiani nel tardo Settecento, Firenze, Olschki, 1996, p. 531-549. 22.Cf. Anna Maria RAO, « Introduzione », dans Anna Maria Rao (dir.), Editoria e cultura a Napoli nel XVIII secolo, Atti del Convegno organizzato dall'Istituto Universitario Orientale, dalla Società Italiana di Studi sul Secolo XVIII e dall'Istituto Italiano per gli Studi filosofici, Napoli 5-7 dicembre 1996, Napoli, Liguori, 1998, p. 3-55.

23. Numéro 19 du 13 avril 1799 : cf. Mario BATTAGLINI (dir.), Il Monitore napoletano 1799, Napoli, Alfredo Guida, 1999, p. 444.

24.Selon une déposition rendue par Filippo Maria Guidi : cf. Annarita BUTTAFUoco, « Eleonora Fonseca Pimentel : una donna nella Rivoluzione », op. cit., p. 59-60.

25. Componimenti poetici Per le leggi date alla nuova Popolazione di Santo Leucio da Ferdinando IV Re delle Sicilie. Cf. Benedetto CROCE, op. cit., p. 14-15 ; Elena URGNANI, op. cit., p. 97-99.

26. Niun diritto compete al Sommo Pontefice sul Regno di Napoli (1790) : cf. Benedetto CROCE, op. cit., p. 19-22 ; Elena URGNANI, op. cit., p. 201-216.

27. Coupable cette dernière d'avoir contribué à dévoiler une conjuration aristocratique contre la République.

28.Sur le rôle des femmes journalistes pendant le «triennio » républicain, cf. Elisa STRUMIA, « Un giornale per le donne nel Piemonte del 1799. "La Vera Repubblicana” », Studi storici, 1989, 4, p. 917-946 ; Laura PISANO, « Giornalismo politico delle donne italiane dalle Repubbliche giacobine all'Unità », dans Laura PISANO, Christiane VEAUVY (dir.), Parole inascoltate. Le donne e la costruzione dello Stato-nazione in Italia e in Francia (1789-1860). Testi e documenti, prefazione di Ginevra CONTI ODORISIO, Roma, Editori Riuniti, 1994, p. 9-77 ; Paroles oubliées: les femmes et la construction de l'État nation en France et en Italie, 1789-1860, préface de Michelle PERROT, Paris, A. Colin, 1997 ; Laura PISANO, « La Nazione al femminile. Aspetti del giornalismo politico tra Settecento e Ottocento in Italia e in Francia », Prometeo, XII, vol. XLVI, 1994, p. 26-33.

29.Cf. Clementina GILY REDA, « As mulheres da revolução napolitana de 1799 : Leonor da Fonseca Pimentel protocientista da comunicação », dans Teresa SANTOS, Sara Marques PEREIRA (dir.), Leonor da Fonseca Pimentel. A portuguesa de Nápoles, op. cit., p. 47-68.

30.Cf. Mario BATTAGLini (dir.), Il Monitore napoletano, op. cit., p. 137 : « Questa parte del Popolo, la quale per fintanto che una miglior istruzione non l'innalzi alla vera dignità di Popolo, bisognerà continuare a chiamar plebe, comprende non solo la numerosa minuta popolazione della città, ma benanche l'altra più rispettabile delle campagne: e 
se sopra di questa parte posa pur nelle monarchie la forza dello Stato, vi posa nella Democrazia non solo la forza ma la dignità. Una gran linea di separazione e forse maggiore, che in qualunque altro luogo disgiunge fra noi questa parte dal rimanente del Popolo, appunto perché non si ha con essa un linguaggio comune. Se ben si rimonti alla cagione de' nostri ultimi mali, si vedranno derivati particolarmente da questa separazione [...] finché dunque la plebe mercé lo stabilimento di una educazione Nazionale non si riduca a pensar come Popolo, conviene, che il Popolo si pieghi a parlar come plebe [...] commischiarsi fra lei ».

31.Vincenzo Cuoco, Histoire de la Révolution de Naples, op. cit., p. 159 ; Vincenzo Cuoco, Saggio storico, op. cit., p. 154.

32.Sur ce problème, cf. Luciano GUERCI, Istruire nelle verità repubblicane. La letteratura politica per il popolo nell'Italia in rivoluzione (1796-1799), Bologna, Il Mulino, 1999.

33.Il Monitore napoletano, op. cit., p. 270.

34.Sur la question de la langue et la tentative de résoudre la difficulté du bilinguisme entre l'italien et les dialectes, par E. Pimentel, cf. Vittorio CRISCUOLO, « Per uno studio della dimensione politica della questione della lingua: settecento e giacobinismo italiano ", Critica storica, XV , 1978, fs. 2-3, p. 305-309, selon lequel il manque une vision positive du dialecte.

35.Rita LIBRANDI, « La comunicazione con la plebe : varietà linguistiche e strategie retoriche nelle "parlate" dei giacobini napoletani », dans Anna Maria RAO (dir.), Napoli 1799. Fra storia e storiografia, Atti del Convegno internazionale, Napoli, 21-24 gennaio 1999, Napoli, Vivarium, 2002, p. 471-492.

36.Beatrice ALFONZETTI, Teatro e tremuoto. Gli anni napoletani di Francesco Saverio Salfi, 1787-1794, Milano, Angeli, 1994 ; Id., « Teatro e rivoluzione: il caso napoletano », dans Anna Maria RAo (dir.), Napoli 1799. Fra storia e storiografia, op. cit., p. 493-517.

37.Il Monitore napoletano, op. cit., p. 138 (9 février).

38.Ibid., p. 178 (16 février).

39.Cf. Anna Maria RAO, « Popular Societies in the Neapolitan Republic of 1799 ", Journal of Modern Italian Studies, 4, 1999, 3, p. 358-369 et Id., « Praticar a democracia : o caso napoletano ", dans Teresa SANTOS, Sara MARQUeS PEREIRA (dir.), Leonor da Fonseca Pimentel. A portuguesa de Nápoles, op. cit., p. 27-45.

40.Supplément au numéro du 5 février, Il Monitore napoletano, op. cit., p. 131-132.

41.Numéro du 9 mai, ibid., p. 540.

42.Ibid., p. 541.

43.Cf. Anna Maria RAO, La Repubblica napoletana del 1799, Roma, Newton \& Compton, 1997, p. 36-39.

44.Cf. par exemple, Laura PISANo, « Il problema del consenso nell'Italia in Rivoluzione, 1796-1799. Le donne al governo della "rigenerazione morale" ", dans Luciano CARTA, Giovanni MURGIA (dir.), Francia e Italia negli anni della Rivoluzione : dallo sbarco francese a Quartu all'insurrezione cagliaritana del 28 aprile 1794, Atti del convegno internazionale di studi (Quartu Sant'Elena-Cagliari, 28-30 aprile 1994), Roma-Bari, Laterza, 1996, p. 288-299 : 298. Plus en général pour le débat sur le rôle des femmes pendant le triennio républicain italien, cf. Luisa RICALDONE, « Il dibattito sulla donna nella letteratura patriottica del triennio (1796-1799) ", Italienische Studien, VII, 1984, p. 23-46 ; Stefano NUTINI, « La « Lettera di una giovane cisalpina alla moglie del generale Bonaparte di A.M. Caronni », Il Risorgimento, XXXVII, 1985, p. 233-244 ; Elisa STRUMIA, «I diritti politici delle donne. L'opinione dei giacobini italiani », dans Robespierre and Co., III seminario 
internazionale (Bagni di Lucca 5-7 novembre 1987), Bologna, Clueb, 1988, 2 vol., I, p. 431-467 ; Annarita BuTTAFUoco, « Straniere in patria. Temi e momenti dell'emancipazione femminile italiana dalle repubbliche giacobine al fascismo », dans Anna Maria CRISPINO (dir.), Esperienza storica femminile nell'età moderna e contemporanea, 2 vol., Roma, Unione donne italiane-Circolo « La Goccia », 1988-1989, I, p. 91-123; Annarita BUTTAFUOCO, "Virtù civiche e virtù domestiche », dans Giuseppina BENASSATI, Lauro Rossi (dir.), L'Italia nella Rivoluzione 1789-1799, catalogo della mostra di Roma, Biblioteca Nazionale Centrale (6 marzo-7 aprile 1990), Bologna, Grafis Edizioni, 1990, p. 81-88 ; Fiamma LUSSANA, « Il concetto di eguaglianza e il dibattito sulla donna nella Francia prerivoluzionaria e in Italia », in Studi storici, 1990, p. 437-455 ; Roberto BoNINI, L'educazione femminile dal privato al pubblico. La partecipazione delle donne ai circoli costituzionali giacobini (1796-1799), quattro saggi con le fonti, Bologna, Clueb, 2001 ; Annarita ButTAFUoco, « La causa delle donne. Cittadinanza e genere nel triennio "giacobino“ in Italia », dans Annarita ButTAFuoco (dir.), Modi di essere: studi, riflessioni interventi sulla cultura e la politica delle donne in onore di Elvira Badaracco, Bologna, EM ricerche, 1991, p. 79-106 ; Rachele FARINA, « De la patrie des italiennes. La voix des femmes à la barre des clubs jacobins ", dans Marie France BRIVE (dir.), Les Femmes et la Révolution française, Actes du colloque international (Toulouse 12-14 avril), 3 vol., Toulouse, Presses universitaires du Mirail, 1989-1991, III, L'effet 89, 1991, p. 51-58 ; Anna Maria RAO, «Il sapere velato. L'educazione delle donne nel dibattito italiano di fine Settecento », dans Andrea Milano (dir.), Misoginia. La donna vista e malvista nella cultura occidentale, Roma, Ed. Dehoniane, 1992, p. 243-310.

\section{RÉSUMÉS}

Alors qu'en Italie, elle fait figure d'héroïne classique, Eleonora de Fonseca Pimentel est restée pratiquement inconnue des historiens français. Pourtant, l'intrépide rédactrice du Moniteur (février-juin 1799) qui, pendant la République de Naples avait assuré une activité fondatrice de journaliste politique - ce qui lui valut d'être exécutée -, fut l'objet de récits mémorables. L'article retrace les divers moments historiographiques qui selon les préférences de leurs auteurs, infléchirent l'exposé de son action. Le contenu de ses idées politiques, axé sur la diffusion des principes républicains dans le peuple, prolonge ses ouvrages littéraires de femme des Lumières que salua Voltaire. Son silence sur la participation politique des femmes reste un sujet à interprétation.

Anna Maria Rao, Eleonora de Fonseca Pimentel, the Monitore Napoletano and the Problem of Political Participation. Whereas in Italy, she is considered a classical heroine, Eléonora de Fonseca Pimentel has remained practically unknown to French historians. Yet this intrepid editor of the Moniteur (February-June 1799), who during the Republic of Naples played a founding role in political journalism - an activity that resulted in her execution - has been the subject of memorable accounts. This article traces the different historiographic moments which according to the preference of their authors, emphasize different aspects of her life. The substance of her political ideas, centered on the dissemination of republican principles, continue the literary 
writings of women of the Enlightenment so praised by Voltaire. Her silence about the political participation of women remains a subject of debate.

INDEX

Mots-clés : peuple, patriotes, Cuoco, Monitore, République napolitaine, Croce

\section{AUTEUR}

ANNA-MARIA RAO

Université de Naples - Federico II, Dipartimento di Discipline storiche, Via Marina 33, 80133 Naples - Italie, annamrao@unina.it 